\title{
Entomología forense: el ciclo de vida de la mosca verde Phaenicia eximia (Wiedemann) (Diptera: Calliphoridae), como herramienta para estimar el intervalo post-mortem
}

\author{
Enio B. Cano \\ Museo de Historia Natural, Escuela de Biología, Universidad de San Carlos de Guatemala, Guatemala y Laboratorio de \\ Entomología Sistemática, Universidad del Valle de Guatemala, Guatemala.
}

*Autor al que se dirige la correspondencia: ecano2005@gmail.com

Recibido: 02 de mayo 2016 / Revisión: 25 de octubre 2015 / Aceptado: 25 de noviembre 2016 / Disponible en línea: 13 de marzo 2017

\section{Resumen}

Q e estudió el ciclo de vida de la mosca verde Phaenicia eximia (Wiedemann) en condiciones controladas de laboratorio a $26^{\circ} \mathrm{C}$, con una humedad relativa de $75 \%$ y un período de iluminación de $12 \mathrm{~h}$ de luz y $12 \mathrm{~h}$ de oscuridad. El tiempo promedio de desarrollo estimado desde la oviposición hasta la salida de los adultos fue de 306 h (una media de casi 13 días). Bajo estas condiciones de temperatura y humedad, los huevos duran cerca de 19 h (0.8 días), las larvas duran unas 170 h (7.1 días) y las pupas unas 116 h (4.8 días). En los meses secos y fríos de noviembre y diciembre en condiciones naturales, el ciclo de vida empírico fue de 25 días, implicando que las bajas temperaturas ralentizan el crecimiento y las altas lo aceleran. Se discute acerca de la importancia del ciclo de vida en la estimación del intervalo post-mortem en casos de muertes violentas en Guatemala.

Palabras claves: Guatemala, larvas, pupas, cadáver, criminalística.

\begin{abstract}
$\mathrm{U}$ nder controlled laboratory conditions, at $26^{\circ} \mathrm{C} 75 \%$ relative humidity and a light/darkness cycle of $12 / 12$ hours, the life cycle of the green bottle fly Phaenicia eximia (Wiedemann) was studied. The estimated average time from oviposition to the emergence of adults was $306 \mathrm{~h}$ (an average of almost 13 days). The eggs stadium is 19 $\mathrm{h}(0.8 \mathrm{~d})$, the larval stage is completed in $170 \mathrm{~h}(7.1 \mathrm{~d})$ and the pupal stage is completed in $116 \mathrm{~h}(4.8 \mathrm{~d})$. Under natural conditions, in the dry and cold months of November and December, the observed life cycle was 25 days, implying that the growth is slow at lower temperatures and faster at higher temperatures. The importance of the life cycle in the estimation of post-mortem interval in cases of violent deaths in Guatemala is discussed.
\end{abstract}




\section{Introducción}

La entomología forense es la aplicación del estudio de los insectos y otros artrópodos en asuntos legales, especialmente en el litigio y el proceso penal relacionado a muertes violentas (e.g., Catts \& Goff, 1992; Greenberg, 1991). El documento más antiguo que muestra la importancia de la entomología forense en la investigación criminal fue escrito en China por Sung Tzu en 1235 (Catts \& Goff, 1992; Greenberg, 1991), en el cual se describe el caso de un homicidio en el que apareció un labrador degollado por una hoz. Para resolver el caso hicieron que todos los labradores de la zona que podían encontrarse relacionados con el muerto, depositasen sus hoces en el suelo, al aire libre, observando que tan solo a una de ellas acudían las moscas y se posaban sobre su hoja, lo que llevó a la conclusión de que el dueño de dicha hoz debía ser el asesino, pues las moscas eran atraídas por los restos de sangre que habían quedado adheridos al "arma" del crimen (Greenberg, 1991). El caso concluyó con la confesión del criminal (Catts \& Goff, 1992).

En Guatemala fue el Dr. Carlos Federico Mora el primero en llamar la atención acerca de los insectos de los cadáveres. Dice Mora (1931, pp. 30-31, 35) que "en Guatemala es común encontrar cadáveres engusanados con larvas de dípteros 24 horas después de la muerte, sobre todo cuando se trata de muertos abandonados a la intemperie... la clase de larvas o de insectos que se encuentra sobre el cadáver, da algunos indicios que permiten apreciar el tiempo [desde la muerte]". Sin embargo, Mora (1931, p. 35) concluyó prematuramente que "numerosas observaciones llevadas a cabo posteriormente, han podido demostrar lo aleatorio de estos cálculos, y han enseñado que las clasificaciones de insectos, no dan resultados concluyentes sino en casos de excepción". Esta conclusión tan radical por parte de la máxima autoridad forense que haya conocido este país, probablemente haya llevado a que los investigadores criminalísticos guatemaltecos le presten muy poca o ninguna atención a la entomofauna cadavérica, considerando a los insectos como contaminantes del cadáver $\mathrm{y}$ en muchos casos nombrando peyorativamente a las agregaciones de larvas de moscas como "arroz", o en el caso específico de Chrysomya rufifacies (Macquart), como "arroz chino" (obs. pers.). En consecuencia, actualmente no se recolectan como indicios ni se utilizan como elementos de prueba en el proceso penal.

Sin embargo, los insectos necrofílicos (aquellos atraídos a los cadáveres) y necrófagos (aquellos que se alimentan de cadáveres) son actualmente muy útiles en la ciencia forense. Cada especie tiene un ciclo de vida de duración casi constante y coloniza un cuerpo por un limitado período de tiempo, con lo cual es posible estimar el tiempo transcurrido desde que el individuo murió (intervalo post-mortem) (Anderson, 2001; Benecke, 1998; Bornemissza, 1957; Catts \& Goff, 1992). Además, los insectos también pueden corroborar si el cuerpo ha sido movido tanto de dentro de una casa hacia el campo, si ha sido trasladado de una región geográfica a otra (Catts \& Goff, 1992), informarnos acerca de las condiciones de higiene (e.g. Benecke \& Lessing, 2001; Sanford, Whitworth, \& Phatak, 2014), abuso de drogas (Goff, Brown, Hewadikaram, \& Omori, 1991; Goff, Brown, \& Omori, 1992; Goff, Omori, \& Goodbrod, 1989) o envenenamiento (Gunatilake \& Goff, 1989; Mahat, Yin, \& Jayaprakash, 2014; Mahat, Zafarina, \& Jayaprakash, 2009; Nuorteva \& Nuorteva, 1982; Patil, Narladkar, \& Rajurkar, 2013) antes de la muerte. La teoría que sostiene la estimación del intervalo post-mortem en entomología forense es la siguiente: inmediatamente después de la muerte comienza la descomposición de los tejidos suaves y la consecuente liberación de compuestos cadavéricos volátiles como alcoholes, ácidos, cetonas, aldehídos, ésteres, compuestos de sulfuro y de nitrógeno, así como de compuestos fenólicos e hidrocarburos (Dekeirsschieter, Stefanuto, Brasseur, Haubruge, \& Focant, 2012). Pero son dos compuestos, el disulfuro de dimetilo (IUPAC = metildisulfonilmetano) y el butan-1-ol, los que se ha demostrado que provocan respuestas olfativas en las antenas de las moscas hembras de la familia Calliphoridae (Frederickx, Dekeirsschieter, Verheggen, \& Haubruge, 2012; Paczkowski, Nicke, Ziegenhagen, \& Schütz, 2015). Así, las moscas hembras grávidas (Wall \& Warnes, 1994) pueden detectar el cuerpo entre 1-3 h después de la muerte (Villet, Richards, \& Midgley, 2010, p. 112). Al llegar al cadáver las moscas colocan sus huevos con lo cual inicia un ciclo biológico que finaliza con la salida de los adultos de las pupas. Así, la estimación de la edad de las moscas permite estimar el tiempo mínimo transcurrido desde la muerte del individuo.

Los insectos son de sangre fría y dependen de la temperatura ambiental para su desarrollo, por consiguiente, la velocidad de desarrollo dependerá de los valores de temperatura ambiental que ocurrieron entre la muerte del individuo ( $\approx$ oviposición de las moscas) y el momento en que se localizaron los insectos en el cuerpo. Así, para dar el siguiente paso -la estimación del intervalo post mortem-, se utiliza el concepto de grados-día acumulados ("accumulated degree days", 
ADD, por sus siglas en inglés, el número de unidades termales que se necesitan para el crecimiento de un insecto en un día) o de grados-hora acumulados ("accumulated degree hours", ADH, las mismas unidades termales medidas cada hora). Esas unidades termales se miden en función de curvas de desarrollo de las moscas a diferentes temperaturas, incluyendo las mínimas y máximas de que permiten el desarrollo. Entonces, para calibrar los modelos de acumulación termal de las moscas de importancia forense en Guatemala, es necesario conocer los ciclos de vida de cada especie en condiciones estandarizadas.

Una de las especies de mosca verde más comunes en Guatemala, al menos antes de la introducción de la especie asiática-africana C. rufifacies por el año 1979 (Schuster \& Cano, 2015), era Phaenicia eximia (Wiedemann). Actualmente se encuentra restringida a hábitats templados y boscosos, sitios en los que con relativa frecuencia se encuentran cadáveres humanos producto de la violencia. Por tanto, el estudio de los ciclos de vida de $P$. eximia, una especie común en cadáveres humanos en Guatemala, es de gran relevancia para que pueda aportar información al momento de ser considerada como indicio y elemento de prueba en el proceso penal. Así, el objetivo de este estudio es describir el ciclo de vida de $P$. eximia en condiciones de temperatura y humedad constante, a fin de que pueda ser utilizada para estimar el intervalo post mortem en procesos médico-legales. Actualmente, el intervalo post mortem es estimado por médicos forenses basados exclusivamente en el aspecto del cadáver.

\section{Materiales y métodos}

\section{Recolecta de huevos, crianza de los estadios y toma de muestras}

Para detectar la presencia de la P. eximia, se colocó en 20 vasos plásticos de $1 \mathrm{~L}, 100 \mathrm{~g}$ de hígado de res fresco, en un bosque fragmentado con cobertura de encinos y pinos, en la ciudad de Guatemala, a una altitud de 1,510 msnm, entre las 08:00 y las 09:00 h. En ese periodo, se encontraron moscas adultas hembras colocando sus masas de huevos (Figura 1). Las moscas adultas fueron recolectadas e identificadas por el autor como $P$. eximia (= Lucilia eximia) la única especie visitante en ese sitio. Así, al día siguiente, a la misma hora, se colocaron nuevas trampas con hígado fresco, las cuales se revisaron $30 \mathrm{~min}$ después y se recolectaron las masas de huevos (junto con el hígado) inmediatamente después de la oviposición, colocándolas en un único recipiente de plástico en cuyo fondo se habían agregado $5 \mathrm{~cm}$ de arena de río seca, tamizada y esterilizada durante $5 \mathrm{~min}$ en horno de microondas. Ese recipiente transparente medía 19 × 33 x $10 \mathrm{~cm}$, con una tapadera plástica a presión a la cual se le realizó una abertura cuadrada de $20 \times 12 \mathrm{~cm}$, en donde se pegó con silicona una malla de tul. La muestra así preparada, se introdujo en una incubadora Percival Scientific $®$, a $26^{\circ} \mathrm{C}$, una humedad relativa de $75 \%$ y un período de iluminación ajustado a $12 \mathrm{~h}$ de luz y $12 \mathrm{~h}$ de oscuridad. Cada día, a las 09:00 y a las 17:00 h se recolectaron 25 especímenes seleccionados aleatoriamente, excepto las pupas de las cuales solo se tomó un espécimen, hasta que emergieron los adultos masivamente. Cuando el $10 \%$ de las larvas puparon se consideró que la fase de pupa se había iniciado y luego se continuó revisando todos los días a las mismas horas para detectar la salida de los adultos, terminado el experimento cuando el 10\%

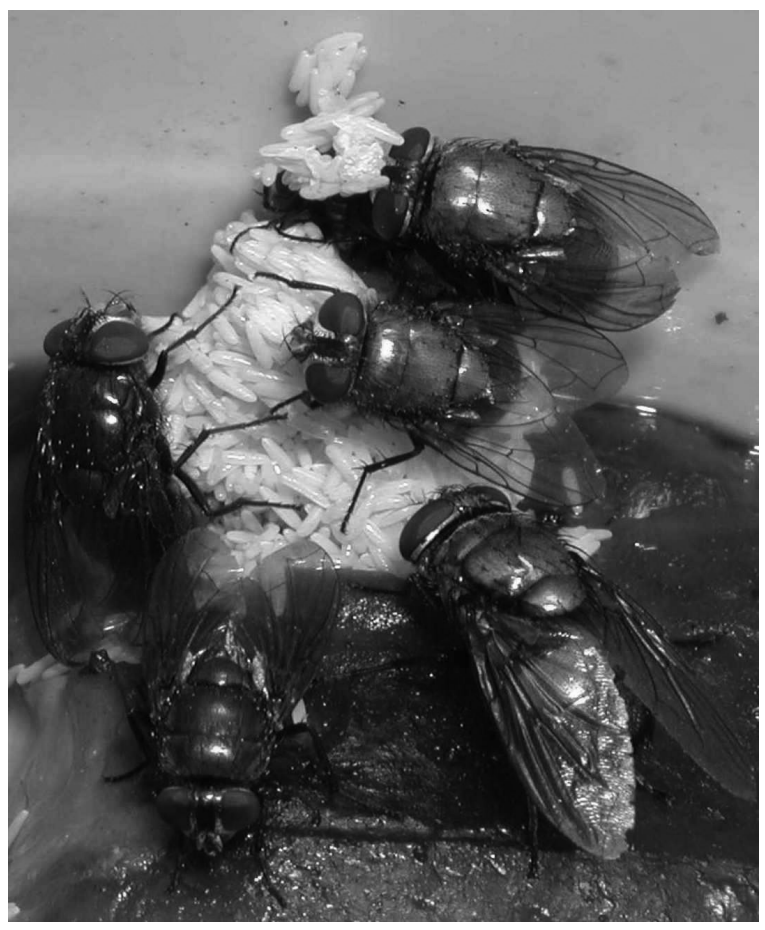

Figura 1. Moscas Phaenicia eximia (Wiedemann) colocando sus masas de huevos en el hígado de res fresco. Como se trata de especímenes hembras, todos tienen los ojos ampliamente separados (los ojos están juntos en los machos). Fotografía en laboratorio. 
de los adultos había emergido. El material recolectado fue fijado en solución de Khale y etiquetado; las moscas adultas se montaron en alfileres y etiquetaron con los datos de colecta; en el mismo alfiler, en puntas de cartulina libre de ácido, se colocaron los restos de las pupas correspondientes. A fin de evitar que la competencia fuera un impedimento para el crecimiento de las larvas (Levot, Brown, \& Shipp, 1979), se agregó hígado fresco extra.

\section{Mediciones y análisis de datos}

Todos los especímenes fijados fueron medidos en su longitud utilizando un calibrador vernier; sin embargo, debido al deterioro de parte del material, no fue posible presentar los datos de todos los individuos. Debido a que el estado de pupa presentó alta uniformidad en su tamaño, únicamente se midió un ejemplar para cada evento de colecta. La curva del ciclo de vida de $P$. eximia se realizó con el programa SPSS 12.0, usando la media y dos desviaciones estándar de las medidas tomadas.

\section{Resultados}

\section{Ciclo de vida}

La Figura 2 muestra los cambios en el tamaño de las larvas de $P$. eximia en función de la edad como un modelo parcialmente sigmoide, con el período de más rápido crecimiento ocurriendo entre las 27 y las $50 \mathrm{~h}$ acumuladas y un período de reducción de tamaño cerca de las $68 \mathrm{~h}$ acumuladas, para luego pasar al estado quiescente de pupa, cuya longitud se asume que ya no varía. Así, bajo condiciones controladas de $26^{\circ} \mathrm{C}$ y $75 \%$ de humedad relativa, con suficiente alimento, encontramos que el ciclo de vida de $P$. eximia desde la oviposición hasta la salida de los primeros adultos dura en promedio $306 \mathrm{~h}$ (Figura 2), aproximadamente 13 días. Los huevos duran unas $19 \mathrm{~h}$, las larvas duran unas $170 \mathrm{~h}$ (aproximadamente 7 días) y las pupas unas $116 \mathrm{~h}$ (aproximadamente 4.85 días). Estos resultados tienen implicaciones en la ciencia forense para determinar el intervalo post mortem, debido a que, si se toman las medidas de las larvas $( \pm 2 \mathrm{DE})$ encontradas en los cadáveres, de acuerdo a la gráfica de la Figura 2 se puede establecer aproximadamente el tiempo mínimo en horas que llevan las larvas en el cuerpo.

\section{Desarrollo de las larvas y pupas}

Las larvas de P. eximia (Figura 3) pasan por tres estadios, caracterizándose las larvas de la última fase del tercer estadio por no alimentarse (larvas postalimentadas) y ser migratorias (Figura 4), abandonando el cadáver o la fuente de alimento y caminando varios metros, hasta que finalmente se entierran y comienzan a encogerse de tamaño (Figura 5), estadio al que llamamos prepupa. Un valor extremo de reducción de tamaño producto de la toma de muestras al azar, se encontró cuando habían transcurrido $118 \mathrm{~h}$ acumuladas. Posteriormente, ocurre el proceso denominado pupariación, en el cual la última exuvia larval queda adherida al resto de la pupa, pasando estas por varios estadios de madurez, como se observa en la Figura 6. Los puparios más jóvenes son de color anaranjado-blanquecino, tornándose anaranjadas con el paso del tiempo, hasta que finalmente se ponen de color café-rojizo muy oscuro. Como los adultos al emerger, destruyen parte de la pupa, estos restos o exuvias pupales (Figura 6) son de gran importancia en entomología forense porque implica el ciclo de vida completo del insecto, desde que las hembras pusieron los huevos hasta que emergió la mosca adulta (Figura 7).

\section{Discusión}

Antes de 1980, P. eximia era la especie de mosca verde más ampliamente distribuida en el país; era la especie verde más común en las carnicerías de los mercados cantonales. Sin embargo, con la introducción de la especie africano-asiática C. rufifacies, por 1979-1980, su distribución se comenzó a restringir. Actualmente es más fácil encontrarla en hábitat de bosque, en donde $C$. rufifacies penetra menos (obs. pers.).

La curva obtenida en este estudio presenta la longitud de los estadios de P. eximia en el tiempo, y puede considerarse como modelo preliminar para establecer el intervalo post mortem. Para esta especie, grosso modo, se resume de la siguiente forma: (a) la detección de huevos no eclosionados representaría un IPM aproximado menor o igual a $19 \mathrm{~h}$, (b) el aparecimiento de la primera generación de larvas ocurre aproximadamente a las 19 $\mathrm{h}$, mientras que el estadio de las larvas más viejas de la primera generación concluye aproximadamente a las 170 h (0.8 a 7.1 días), así, la longitud de las larvas $( \pm 2 \mathrm{DE})$ puede usarse para estimar con cierta precisión el IPM (e.g. una larva de $10 \mathrm{~mm}( \pm 2 \mathrm{DE})$ se presenta cerca de las $90 \mathrm{~h}$ a $26^{\circ} \mathrm{C}$ ), (c) las pupas de la primera 


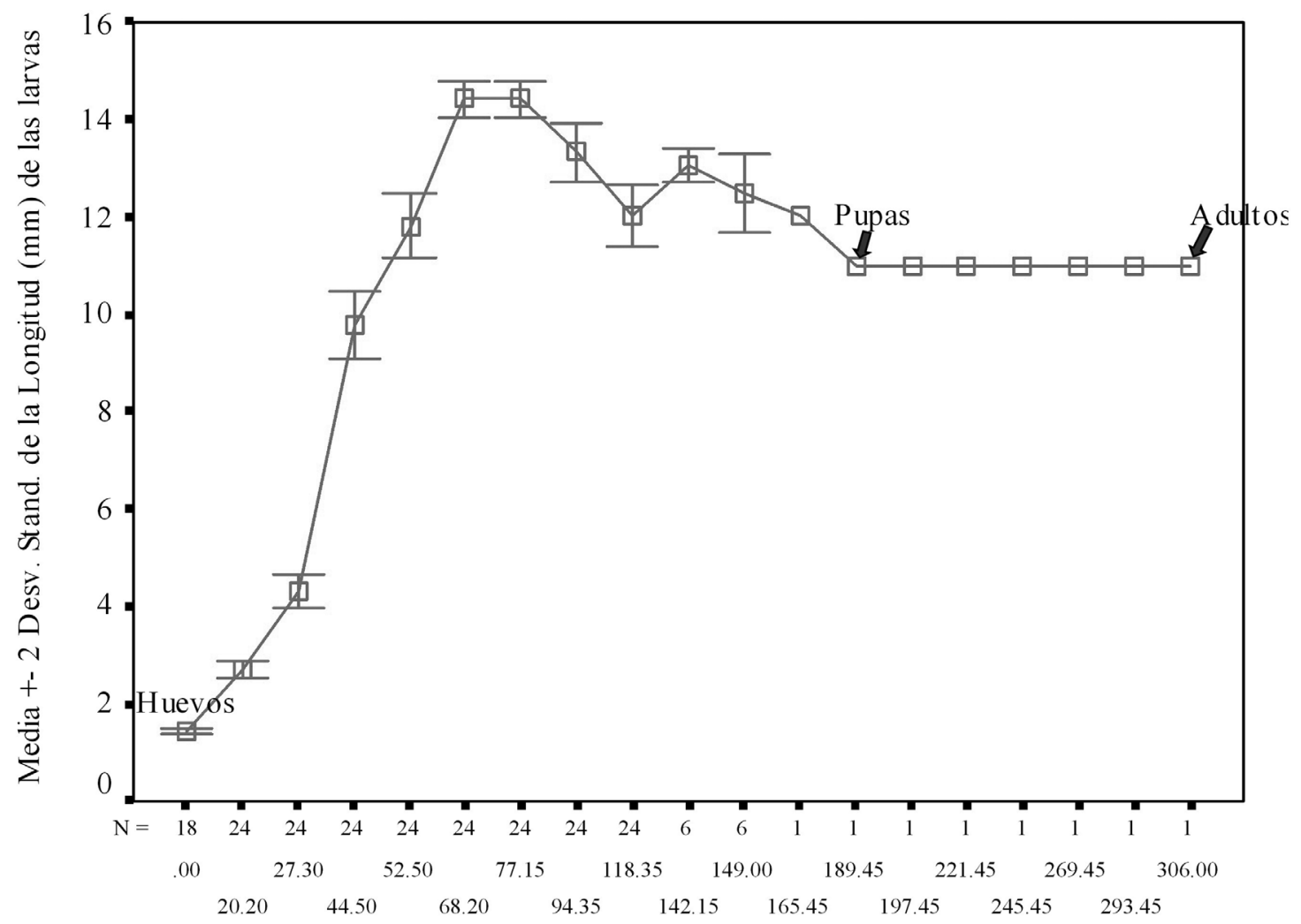

Número de horas acumuladas

Figura 2. Cambios en el tamaño de las larvas de Phaenicia eximia en función del tiempo transcurrido (horas), en condiciones de laboratorio $\left(\mathrm{t}=26^{\circ} \mathrm{C}, \mathrm{HR}=75 \%\right)$.

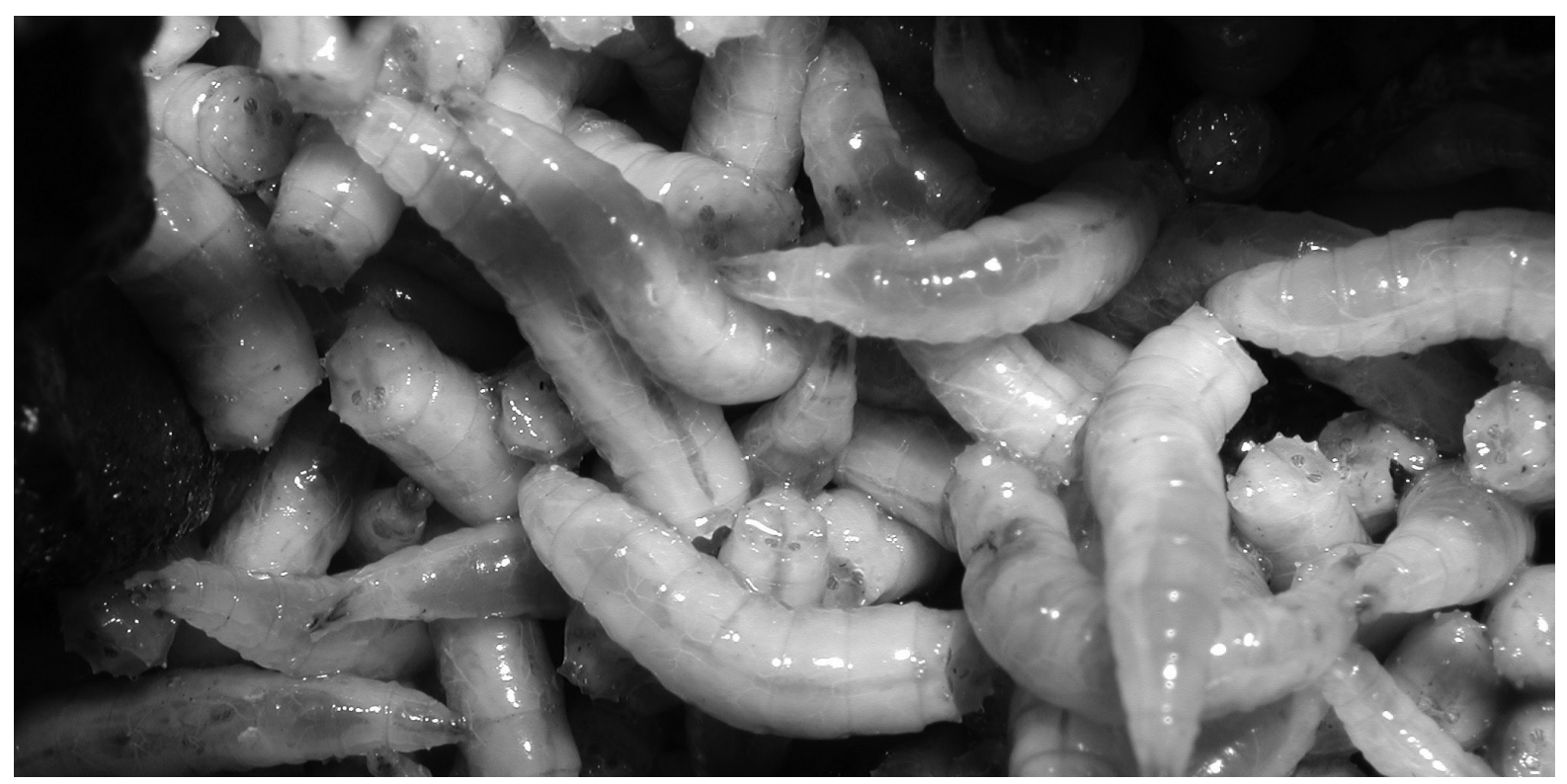

Figura 3. Larvas del tercer estadio de Phaenicia eximia. 


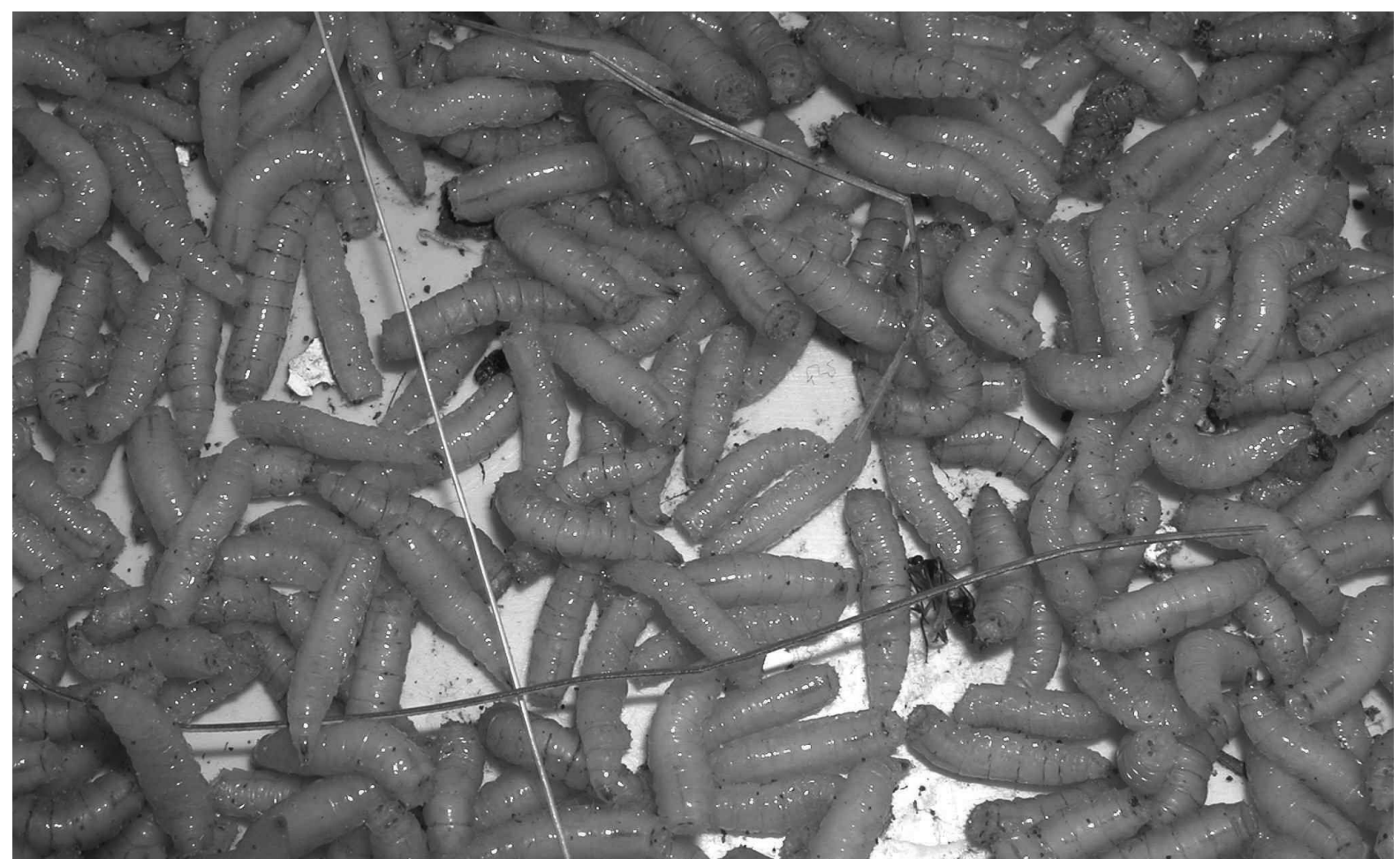

Figura 4. Larvas migrantes de Phaenicia eximia. Fotografía tomada en el laboratorio.

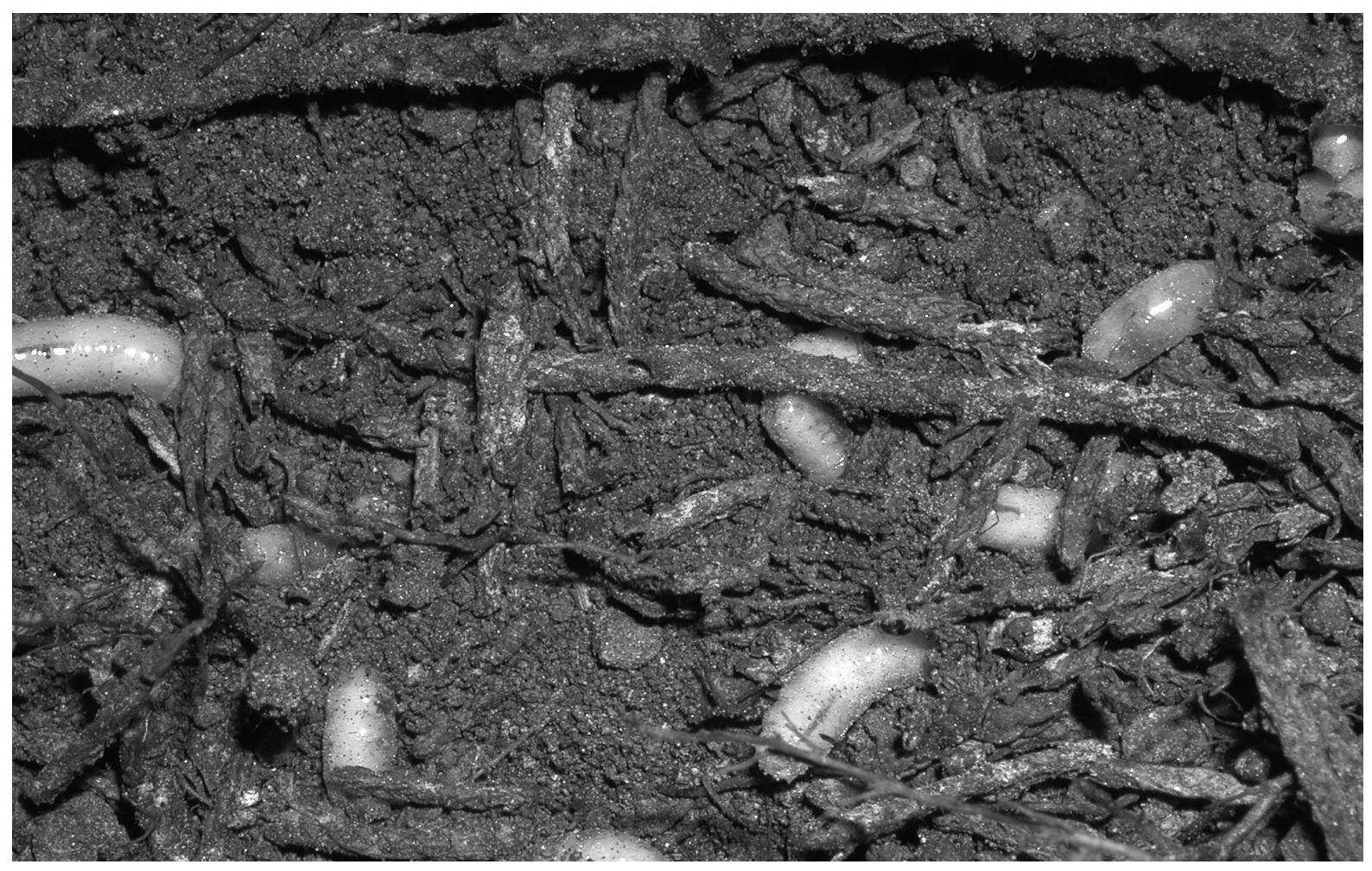

Figura 5. Larvas migrantes, escondiéndose en el suelo (prepupas). Fotografía tomada en el campo. 


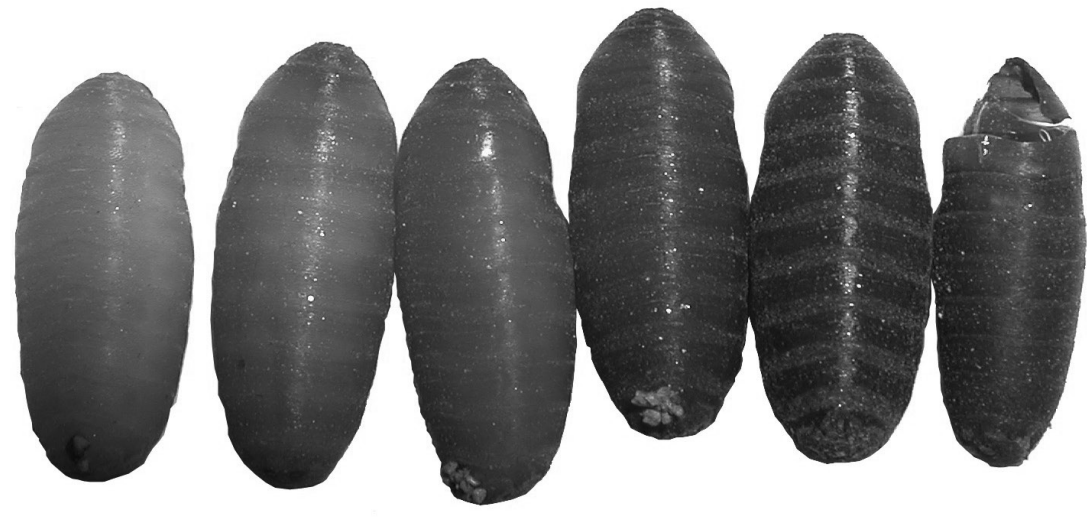

Figura 6. Diferentes estados pupales (puparios). Nota las diferencias en color y al final, la pupa vacía implicando la salida de la mosca adulta.

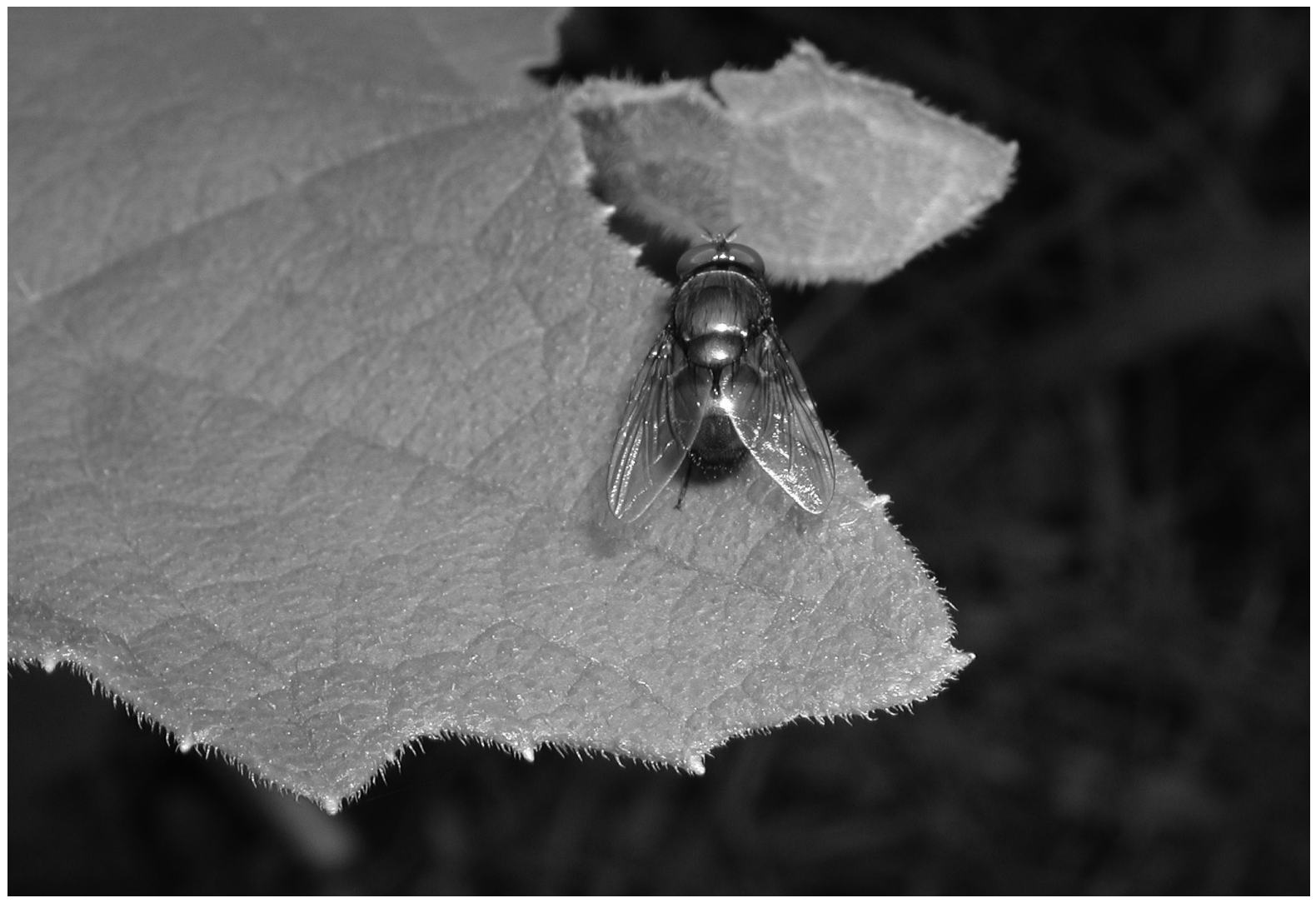

Figura 7. Adulto macho de Phaenicia eximia recién emergido. Fotografía tomada en el campo. 
generación se presentan entre las 170 y 286 h (7.1 a 11.9 días) y (d) los adultos de la primera generación emergen cerca de las 320 h (13.3 días). La Figura 2 muestra que el modelo tiene dos limitaciones: (a) entre las 138 y 170 horas las larvas ya no incrementan linealmente su longitud (de hecho, la longitud se reduce previo a la formación de la pupa), por lo que para las larvas con longitudes entre 12 y $14 \mathrm{~mm}$ sólo es preciso decir que su IPM se ubica entre 138 y 170 h (5.8 a 7.1 días) y (b) estos datos sólo son válidos para la temperatura y humedad reportadas en este estudio. Por otro lado, en experimentos no controlados en el campo se encuentran varias generaciones de moscas, por lo cual, se debe conocer claramente que el material con el que se está trabajando incluye siempre a las larvas más viejas (la primera generación).

Aunque el ciclo de vida documentado en este estudio a $26^{\circ} \mathrm{C}$ duró unos 13 días, en un estudio no publicado en condiciones naturales no controladas, el ciclo de vida de $P$. eximia varió de acuerdo a la temperatura entre 14 y 25 días: a bajas temperaturas (en el bosque, en los meses diciembre-enero) el ciclo de vida tuvo una duración de 25 días, implicando que las bajas temperaturas ralentizan el crecimiento y las altas lo aceleran. Por tanto, es necesario realizar más estudios como el presente, a diferentes temperaturas, para tener curvas estandarizadas de desarrollo con miras a la estimación del intervalo post mortem, determinando las temperaturas mínimas de desarrollo y las tasas de crecimiento termal (horas/grado, días/grado acumulados). Además, debe notarse que el modelo de la Figura 2 está basado en datos recopilados únicamente dos veces por día, y no durante todas las horas sucesivas a lo largo del ciclo de vida. Por tanto, para obtener modelos predictivos que puedan aplicarse a la ciencia forense en Guatemala, es necesario incluir medidas detalladas de todo el ciclo de vida (particularmente de las larvas) con sus correspondientes medidas de confianza (e.g. Baqué, Filmann, Verhoff, \& Amedt, 2015; Wells, Lecheta, Moura, \& LaMotte, 2014).

En cuanto a la relevancia de este estudio, Guatemala es uno de los países más violentos en el mundo y también muy rico en diversidad de insectos, por lo que el uso de insectos necrófagos y necrófilos para la estimación del IPM debería ser validado y empleado como medio científico de prueba. El Instituto Nacional de Ciencias Forenses (Inacif) en el 2016 reportó la realización de 5,875 y 5,677 necropsias con señales de muerte violenta, para los años 2014 y 2015 , lo que representa una tasa anual de 31.2 y 33.84 muertes vio- lentas por cada 100 mil habitantes, respectivamente. Así, enriquecer las ciencias criminalísticas del país con nuevas herramientas que permitan una efectiva persecución penal, es imperativo. Tanto la abundante literatura disponible, como los resultados, sugieren que insectos como la mosca $P$. eximia, de la cual ahora se conoce parte de su ciclo de vida, pueden ser útiles en el proceso penal, como indicios o elementos de prueba.

Existen cuatro razones para el uso de insectos necrófilos y necrófagos en la investigación forense. En primer lugar, son fuente de evidencia como la sangre, las huellas digitales, los pelos, las fibras, las armas, etc; aunque en Guatemala son descartados o limpiados de los cuerpos, con lo cual se pierde información que podría ser útil, poco útil, nada útil o hasta contundente para concluir un caso. En segundo lugar, la fauna de artrópodos coloniza los cadáveres en estados específicos de descomposición, formando ensambles particulares, lo que provee de un buen apoyo para la estimación del intervalo post mortem a muy largo plazo (hasta 40 días, como en Schuster \& Cano, 2005). En tercer lugar, como en el presente estudio, las moscas verdes de la familia Calliphoridae tienen ciclos de vida bastante exactos y constantes, con lo cual, al conocer el tiempo de duración de los ciclos de vida y la temperatura ambiental de desarrollo, podemos estimar el momento en el que el insecto llegó al cadáver. Por último, la entomología forense se ha establecido plenamente en el campo de las ciencias criminalísticas, como lo demuestran los diferentes textos internacionales (e.g. Amendt, Goff, Campobasso, \& Grassberger 2010; Byrd \& Castner, 2009; Rivers \& Dahlem, 2014; Tomberlin \& Benbow, 2015), los laboratorios especializados en el mundo y las múltiples publicaciones de procedimientos estandarizados en revistas de corriente principal (e.g. Adams \& Hall, 2003; Amendt et al., 2007; Byrd \& Castner, 2009; Hall, 2001; Villet et al., 2010).

\section{Agradecimientos}

Agradezco al M.Sc. Javier Rivas, al Dr. Jack Schuster y al Lic. Federico Nave, por los valiosos comentarios que mejoraron sustancialmente la versión final del manuscrito. Este es un aporte al proyecto 36-00 apoyado por el Consejo Nacional de Ciencia y Tecnología de Guatemala (Concyt). 


\section{Referencias}

Adams, Z. J., \& Hall, M. J. (2003). Methods used for the killing and preservation of blowfly larvae, and their effect on post-mortem larval length. Forensic Science International, 138, (1-3) 50-61.

Amendt, J., Campobasso, C. P., Gaudry, E., Reiter, C., LeBlanc, H. N., \& Hall, M. J. (2007). Best practice in forensic entomology-standards and guidelines. International Journal of Legal Medicine, 121(2), 90-104. doi: 10.1007/s00414006-0086-x

Amendt, J., Goff, M. L., Campobasso, C. P., \& Grassberger, M. (2010). Current concepts in forensic entomology. London: Springer.

Anderson, G. S. (2001). Insect succession on carrion and its relationship to determining time of death. En J. H. Byrd \& J. L. Castner (Eds.), Forensic entomology. The utility of arthropods in legal investigations (pp. 143-175). London: CRC Press.

Baqué, M., Filmann, N., Verhoff, M. A., \& Amedt, J. (2015). Establishment of developmental charts for the larvae of the blow fly Calliphora vicina using quantile regression. Forensic Science International, 248, 1-9.

Benecke, M. (1998). Six forensic entomology cases: Description and commentary. Journal of Forensic Sciences, 43, 797-805.

Benecke, M., \& Lessing, R. (2001). Child neglect and forensic entomology. Forensic Science International, 120(1-2), 155-159.

Bornemissza, G. F. (1957). An analysis of arthropod succession in Carrion and the effect of its descomposition on the soil fauna. Australian Journal of Zoology, 5(1), 1-12.

Byrd, J. H., \& Castner, J. L. (2009). Forensic entomology: the utility of arthropods in legal investigations, Second edition. Boca Raton: CRC Press.

Catts, E. P., \& Goff, M. L. (1992). Forensic entomology in criminal investigations. Annual Review of Entomology, 37, 252-272. doi:10.1146/annurev. en.37.010192.001345

Dekeirsschieter, J., Stefanuto, P.-H., Brasseur, C., Haubruge, E., \& Focant, J.-F. (2012). Enhanced characterization of the smell of death by comprehensive two-dimensional gas chromatography time-of-flight mass spectrometry (GCxGC-TOFMS). Plos One, 7(6), e39005. doi: 10.1371/journal.pone.0039005

Frederickx, C., Dekeirsschieter, J., Verheggen, F. J., \& Haubruge, E. (2012). Responses of Lucilia sericata Meigen (Diptera: Calliphoridae) to cadaveric volatile organic compounds. Journal of Forensic Sciences, 57(2), 386-390. doi: 10.1111/j.1556-4029.2011.02010.x.

Goff, M. L., Brown, W. A., Hewadikaram, K. A., \& Omori, A. I. (1991). Effect of heroin in decomposing tissues on the development rate of Boettcherisca peregrina (Diptera, Sarcophagidae) and implications of this effect on estimation of postmortem intervals using arthropod development patterns. Journal of Forensic Sciences, 36(2), 537-542.

Goff, M. L., Brown, W. A., \& Omori, A. I. (1992). Preliminary observations of the effect of methamphetamine in decomposing tissues on the development rate of Parasarcophaga ruficornis (Diptera: Sarcophagidae) and implications of this effect on the estimations of postmortem intervals. Journal of Forensic Sciences, 37(3), 867-872.

Goff, M. L., Omori, A. I., \& Goodbrod, J. R. (1989). Effect of cocaine in tissues on the development rate of Boettcherisca peregrina (Diptera: Sarcophagidae). Journal of Medical Entomology, 26(2), 91-3. doi: 10.1093/jmedent/26.2.91

Greenberg, B. (1991). Flies as forensic indicators. Journal of Medical Entomology, 28(5), 565-577.

Gunatilake, K., \& Goff, M. L. (1989). Detection of organophosphate poisoning in a putrefying body by analysing arthropod larvae. Journal of Forensic Sciences, 34, 714-716.

Hall, D. (2001). The forensic entomologist as expert witness. En J. H. Byrd \& J. L. Castner (Eds.), Forensic entomology. The utility of arthropods in legal investigations (pp. 379-400). Boca Ratón: CRC Press.

Instituto Nacional de Ciencias Forenses. (2016). Necropsias realizadas en las sedes periciales del INACIF, a Nivel Nacional. En Datos Numéricos, Información Anual, Consolidado de solicitud Anual 2016. Guatemala: Autor. Recuperado de 
http://www.inacif.gob.gt/index.php?option=com content\&view $=$ article\&id $=97 \&$ Itemid $=18$

Levot, G. W., Brown K. R., \& Shipp, E. (1979). Larval growth of some calliphorid and sarcophagid Diptera. Bulletin of Entomological Research, 69, 469-475.

Mahat, N. A., Yin, C. L., \& Jayaprakash, P. T. (2014). Influence of Paraquat on Chrysomya megacephala Fabricius (Diptera: Calliphoridae) infesting minced-beef substrates in Kelantan, Malaysia. Journal of Forensic Sciences, 59(2), 529-532. doi: 10.1111/1556-4029.12355

Mahat, N. A, Zafarina, Z., \& Jayaprakash, P. T. (2009). Influence of rain and malathion on the oviposition and development of blowflies (Diptera: Calliphoridae) infesting rabbit carcasses in Kelantan, Malaysia. Forensic Science International, 192, 19-28. doi: 10.1016/j. forsciint.2009.07.008

Mora, C. F. (1931). Manual de medicina forense. Guatemala: Tipografía Nacional.

Nuorteva, P., \& Nuorteva, S. L. (1982). The fate of mercury in sarcosaprophagous flies and in insects eating them. Ambio, 11(1), 34-37.

Paczkowski, S., Nicke, S., Ziegenhagen, H., \& Schütz, S. (2015). Volatile emission of decomposing pig carcasses (Sus scrofa domesticus L.) as an indicator for the Postmortem Interval. Journal of Forensic Sciences, 60(1), 130-137. doi: 10.1111/1556-4029.12638

Patil, V. G., Narladkar, B. W., \& Rajurkar, S. R. (2013). Application of forensic entomology for judging the different poisoning cases in animals: In vitro studies in Wistar rats (Rattus norvegicus). Journal of Veterinary Parasitology, 27(2), 74-78.

Rivers, D. B., \& Dahlem, G. A. (2014). The science of forensic entomology. Oxford: Wiley Blackwell.

Sanford, M. R., Whitworth, T. L., \& Phatak, D. R. (2014). Human wound colonization by Lucilia eximia and Chrysomya rufifacies (Diptera: Calliphoridae): Myiasis, perimortem, or postmortem colonization? Journal of Medical Entomology, 51(3), 716-719.

Schuster, J. C., \& Cano, E. B. (2005). ¿Para qué estudiar insectos? Revista de la Universidad del Valle de Guatemala, 14, 58-63.
Schuster, J. C., \& Cano, E. B. (2015). La importancia científica e histórica de la Colección de Artrópodos de la Universidad del Valle de Guatemala con un inventario de los tipos primarios depositados. Revista de la Universidad del Valle de Guatemala, 31, 11-16.

Tomberlin, J. K., \& Benbow, E. M. (2015). Forensic entomology. International dimensions and frontiers. London: CRC Press.

Villet, M. H., Richards, C. S., \& Midgley, J. M. (2010). Contemporary precision, bias and accuracy of minimum post-mortem intervals estimated using development of carrion-feeding insects. En J. Amendt, M. L. Goff, C. P. Campobasso \& M. Grassberger (Eds.), Current concepts in forensic entomology (pp. 109-137). New York: Springer.

Wall, R., \& Warnes, M. L. (1994). Responses of the sheep blowfly Lucilia sericata to carrion odour and carbon dioxide. Entomologia Experimentalis et Applicata, 73, 239-246.

Wells, J. D., Lecheta, M. C., Moura, M. O., \& LaMotte, L. R. (2014). An evaluation of sampling methods used to produce insect growth models for postmortem interval estimation. International Journal of Legal Medicine, 129(2), 405-410. doi: 10.1007/s00414-014-1029-6. 